\title{
Oscillation of a class of the fourth-order nonlinear difference equations
}

\section{Zuzana Došlá and Jana Krejčová*}

"Correspondence:

krejcovajana@mail.muni.cz

Department of Mathematics and

Statistics, Faculty of Science,

Masaryk University, Brno, Czech Republic

\begin{abstract}
In this article, a class of fourth-order difference equations with quasi-differences and deviating argument is considered. We state a new oscillation theorem for the sublinear case and we complete the existing results in the literature. Our approach is based on considering Equation (1) as a system of the four-dimensional difference system and on the cyclic permutation of the coefficients in the difference equations.
\end{abstract}

\section{Introduction}

In this article, we consider a class of fourth-order nonlinear difference equations of the form

$$
\Delta\left(a_{n}\left(\Delta b_{n}\left(\Delta c_{n}\left(\Delta x_{n}\right)^{\gamma}\right)^{\beta}\right)^{\alpha}\right)+d_{n} x_{n+\tau}^{\lambda}=0
$$

where $\alpha, \beta, \gamma, \lambda$ are the ratios of odd positive integers, $\tau \in \mathbb{Z}$ is a deviating argument and $\left\{a_{n}\right\},\left\{b_{n}\right\},\left\{c_{n}\right\},\left\{d_{n}\right\}$ are positive real sequences defined for $n \in \mathbb{N}_{0}=\left\{n_{0}, n_{0}+1, \ldots\right\}, n_{0}$ is a positive integer, and $\Delta$ is the forward difference operator defined by $\Delta x_{n}=x_{n+1}-x_{n}$.

By a solution of Equation (1) we mean a real sequence $\left\{x_{n}\right\}$ satisfying Equation (1) for $n \in \mathbb{N}_{0}$. A non-trivial solution $\left\{x_{n}\right\}$ of $(1)$ is said to be non-oscillatory if it is either eventually positive or eventually negative, and it is otherwise oscillatory. Equation (1) is said to be oscillatory if all its solutions are oscillatory.

In the last few years, great attention has been paid to the study of fourth-order nonlinear difference equations, see [1-10] and references therein.

If $a_{n}=c_{n}=1, \alpha=\gamma=1$ and $\tau=3$, then (1) takes the form

$$
\Delta^{2}\left(b_{n}\left(\Delta^{2} x_{n}\right)^{\beta}\right)+d_{n} x_{n+3}^{\lambda}=0
$$

The oscillatory and asymptotic properties of solutions of (2) have been investigated in [4, $11,12]$ under the conditions

$$
\sum_{n=n_{0}}^{\infty} \frac{n}{b_{n}^{1 / \beta}}=\infty \text { and } \sum_{n=n_{0}}^{\infty}\left(\frac{n}{b_{n}}\right)^{1 / \beta}=\infty
$$

while articles $[2,10,13]$ deal with cases where at least one of these series is convergent (see also the references therein).

@ 2012 Došlá and Krejčová; licensee Springer. This is an Open Access article distributed under the terms of the Creative Commons Attribution License (http://creativecommons.org/licenses/by/2.0), which permits unrestricted use, distribution, and reproduction in any medium, provided the original work is properly cited. 
Equation (1) is a special case of nonlinear fourth-order equation with deviating argument investigated in the recent articles $[1,2]$. In [1], necessary and sufficient conditions for the oscillation of all bounded solutions of (1) (the so-called B-oscillation) have been given. In [2], oscillation criteria for (1) have been established using the analysis of nonoscillatory solutions and by comparison with certain first- and second-order difference equations.

Equation (1) with $\tau=2$ can be seen as a coupled system of two second-order difference equations of the form

$$
\left\{\begin{array}{l}
\Delta\left(r_{n}\left(\Delta x_{n}\right)^{\alpha}\right)=-\varphi_{n} z_{n+1}^{\eta} \\
\Delta\left(q_{n}\left(\Delta z_{n}\right)^{\beta}\right)=\psi_{n} x_{n+1}^{\lambda}
\end{array}\right.
$$

Indeed, eliminating $z$ from the first equation, this system can be rewritten as

$$
\Delta\left(q_{n+1}\left(\Delta\left(\varphi_{n}^{-1 / \eta}\left(\Delta\left(r_{n}\left(\Delta x_{n}\right)^{\alpha}\right)\right)^{1 / \eta}\right)\right)^{\beta}\right)+\psi_{n+1} x_{n+2}^{\lambda}=0 .
$$

System (3) is a special case of more general coupled systems. Those oscillatory properties have been investigated in [5].

Our approach here is to consider (1) as a four-dimensional system. If

$$
y_{n}=c_{n}\left(\Delta x_{n}\right)^{\gamma}, \quad z_{n}=b_{n}\left(\Delta y_{n}\right)^{\beta}, \quad w_{n}=a_{n}\left(\Delta z_{n}\right)^{\alpha},
$$

then Equation (1) can be written as the nonlinear system

$$
\left\{\begin{array}{l}
\Delta x_{n}=C_{n} y_{n}^{1 / \gamma}, \\
\Delta y_{n}=B_{n} z_{n}^{1 / \beta}, \\
\Delta z_{n}=A_{n} w_{n}^{1 / \alpha}, \\
\Delta w_{n}=-D_{n} x_{n+\tau}^{\lambda},
\end{array}\right.
$$

where

$$
A_{n}=a_{n}^{-1 / \alpha}, \quad B_{n}=b_{n}^{-1 / \beta}, \quad C_{n}=c_{n}^{-1 / \gamma}, \quad D_{n}=d_{n} .
$$

Obviously, if $(x, y, z, w)$ is a solution of system (S) and one of its components is of one sign, then all its components are of one sign.

System (S) can be viewed as a discrete analogue of the four-dimensional differential system investigated by Kusano et al. [14], and by Chanturia [15]. In that article, the oscillation of the $n$-dimensional differential systems was investigated in terms of Property $\mathrm{A}$ (which reads for equations of even order as the oscillation of all solutions) and Property $\mathrm{B}$. Observe that in [16] we have used this approach to study Property B for system (S) assuming $D_{n}<0$.

Motivated by these articles, we study the oscillatory properties of solutions of (1). First we show the influence of the deviating argument $\tau$ on the existence of quickly oscillatory solutions and we describe the so called cyclic permutation for (1). Our main goal is to state a new oscillation theorem for Equation (1) in the sublinear case $\lambda<\alpha \beta \gamma$ and to extend the 
existing oscillation results in the literature in case where the difference operator in (1) is in the canonical form, i.e., when

$$
\sum_{n=n_{0}}^{\infty} a_{n}^{-1 / \alpha}=\sum_{n=n_{0}}^{\infty} b_{n}^{-1 / \beta}=\sum_{n=n_{0}}^{\infty} c_{n}^{-1 / \gamma}=\infty
$$

Our results are based on the conditions for the non-existence of non-oscillatory solutions and on the change of summation for double series. Due to our approach considering (1) as a four-dimensional system, we extend for any $\tau \in \mathbb{Z}$ some results of [2] stated for a delay $\tau \leq 0$. Using cyclic permutation we show how it is possible to extend oscillation criteria to the case when one of the series in $(\mathrm{H})$ is convergent.

\section{Existence of quickly oscillatory solutions}

Prototypes of oscillatory solutions of (1) are solutions of the form

$$
x_{n}=(-1)^{n} p_{n}, \quad p_{n}>0 \text { for } n \in \mathbb{N}_{0} .
$$

Such solutions are called quickly oscillatory and the following result can be seen as a necessary condition for their existence.

Theorem 1 Equation (1) with $\tau$ even has no quickly oscillatory solutions.

Proof Let $x_{n}=(-1)^{n} p_{n}$ be a quickly oscillatory solution of $(1)$. Then

$$
\Delta x_{n}=(-1)^{n+1}\left(p_{n+1}+p_{n}\right) .
$$

From the first equation of system (S) we have

$$
y_{n}=\left(\frac{\Delta x_{n}}{C_{n}}\right)^{\gamma}=(-1)^{n+1} q_{n}
$$

where $q_{n}=\left(\frac{p_{n+1}}{C_{n}}+\frac{p_{n}}{C_{n}}\right)^{\gamma}>0$. From the second equation of (S) we obtain

$$
z_{n}=\left(\frac{\Delta y_{n}}{B_{n}}\right)^{\beta}=(-1)^{n} r_{n}
$$

where $r_{n}=\left(\frac{q_{n+1}}{B_{n}}+\frac{q_{n}}{B_{n}}\right)^{\beta}>0$. Repeating argument, we get from the third equation of (S)

$$
w_{n}=\left(\frac{\Delta z_{n}}{A_{n}}\right)^{\alpha}=(-1)^{n+1} s_{n}
$$

where $s_{n}=\left(\frac{r_{n+1}}{A_{n}}+\frac{r_{n}}{A_{n}}\right)^{\alpha}>0$. Consequently, from here and from the fourth equation we have

$$
\Delta w_{n}=(-1)^{n}\left(s_{n+1}+s_{n}\right)=-D_{n}(-1)^{(n+\tau) \lambda} p_{n+\tau}^{\lambda}=(-1)^{n+1+\tau} D_{n} p_{n+\tau}^{\lambda},
$$

which gives a conclusion.

By the method used in the proof of Theorem 1 we can easily construct equations possessing a quickly oscillatory solution. 
Example 1 Consider the equation

$$
\Delta^{2}\left(\Delta^{2} x_{n}\right)^{\beta}+\frac{3^{2 \beta}\left(2^{\beta}+1\right)^{2}}{2^{\tau \lambda}} 2^{n(\beta-\lambda)} x_{n+\tau}^{\lambda}=0,
$$

where $\tau$ is an odd positive integer. This equation has a quickly oscillatory solution $x_{n}=$ $(-1)^{n} 2^{n}$. Indeed, $p_{n}=2^{n}, q_{n}=2^{n} 3, r_{n}=2^{n \beta} 3^{2 \beta}, s_{n}=2^{n \beta} 3^{2 \beta}\left(2^{\beta}+1\right)$ and the value of $d_{n}$ follows from the relation $d_{n}=\left(s_{n+1}+s_{n}\right) / p_{n+\tau}^{\lambda}$.

If $\beta \geq \lambda$, then Equation (6) has all solutions oscillatory (see, e.g., Proposition 1 below). However, if $\beta<\lambda$, then by [4, Theorems 3.5, 3.6] Equation (6) has also non-oscillatory solutions.

\section{Cyclic permutation}

In this section, we describe the left-ordered cyclic permutation of coefficients in (1).

Lemma 1 The following statements are equivalent:

(i) $x$ is a solution of (1).

(ii) $y=\left\{y_{n}\right\}$, where $y_{n}=c_{n}\left(\Delta x_{n}\right)^{\gamma}$, is a solution of

$$
\Delta\left(\frac{1}{d_{n}^{1 / \lambda}}\left(\Delta a_{n}\left(\Delta b_{n}\left(\Delta y_{n}\right)^{\beta}\right)^{\alpha}\right)^{1 / \lambda}\right)+\frac{1}{c_{n+\tau}^{1 / \gamma}} y_{n+\tau}^{1 / \gamma}=0
$$

(iii) $z=\left\{z_{n}\right\}$, where $z_{n}=b_{n}\left(\Delta y_{n}\right)^{\beta}$, is a solution of

$$
\Delta\left(c_{n+\tau}\left(\Delta \frac{1}{d_{n}^{1 / \lambda}}\left(\Delta a_{n}\left(\Delta z_{n}\right)^{\alpha}\right)^{1 / \lambda}\right)^{\gamma}\right)+\frac{1}{b_{n+\tau}^{1 / \beta}} z_{n+\tau}^{1 / \beta}=0 .
$$

(iv) $w=\left\{w_{n}\right\}$, where $w_{n}=a_{n}\left(\Delta z_{n}\right)^{\alpha}$ is a solution of

$$
\Delta\left(b_{n+\tau}\left(\Delta c_{n+\tau}\left(\Delta \frac{1}{d_{n}}\left(\Delta w_{n}\right)^{1 / \lambda}\right)^{\gamma}\right)^{\beta}\right)+\frac{1}{a_{n+\tau}^{1 / \alpha}} w_{n+\tau}^{1 / \alpha}=0 .
$$

Proof First we prove that (i) is equivalent to (ii). If we express $x$ from the last equation in (S) we obtain

$$
x_{n+\tau}=-\frac{1}{d_{n}^{1 / \lambda}}\left(\Delta w_{n}\right)^{1 / \lambda}=-\frac{1}{d_{n}^{1 / \lambda}}\left(\Delta a_{n}\left(\Delta b_{n}\left(\Delta y_{n}\right)^{\beta}\right)^{\alpha}\right)^{1 / \lambda} .
$$

Thus, from here and the first equation in $(\mathrm{S})$ we have

$$
\Delta x_{n+\tau}=-\Delta\left(\frac{1}{d_{n}^{1 / \lambda}}\left(\Delta a_{n}\left(\Delta b_{n}\left(\Delta y_{n}\right)^{\beta}\right)^{\alpha}\right)^{1 / \lambda}\right)=\frac{1}{c_{n+\tau}^{1 / \gamma}} y_{n+\tau}^{1 / \gamma},
$$

which yields Equation (R1). To prove that (i) is equivalent to (iii) we use the same process. Using (5) and (7) we have

$$
\Delta x_{n}=-\Delta\left(\frac{1}{d_{n-\tau}^{1 / \lambda}}\left(\Delta a_{n-\tau}\left(\Delta z_{n-\tau}\right)^{\alpha}\right)^{1 / \lambda}\right) .
$$


Substituting this into

$$
\Delta y_{n}=\Delta\left(c_{n}\left(\Delta x_{n}\right)^{\gamma}\right)
$$

and using the second equation of (S) we get Equation (R2).

To prove that (i) is equivalent to (iv) we proceed as before, expressing $\Delta z$ in terms of $w$ from the third equation of (S) and from (5) and comparing both expressions.

Theorem 2 Equation (1) is oscillatory if and only if Equation $\left(R_{i}\right)$ is oscillatory for $i \in$ $\{1,2,3\}$.

Remark 1 By Theorem 2 Equation (2) is oscillatory if and only if the equation

$$
\Delta^{2}\left(\frac{1}{d_{n}^{1 / \lambda}}\left(\Delta^{2} z_{n}\right)^{1 / \lambda}\right)+\frac{1}{b_{n+3}^{1 / \beta}} z_{n+3}^{1 / \beta}=0
$$

is oscillatory. Observe that the difference operator in this equation is in the canonical form if $\sum_{n=n_{0}}^{\infty} d_{n}=\infty$.

Remark 2 The cyclic permutation for the coupled system (3) means that equations in (3) are considered in the opposite order. Hence, $(x, z)$ is a solution of (3) if and only if $(u, v)=(-z, x)$ is a solution of the system

$$
\left\{\begin{array}{l}
\Delta\left(q_{n}\left(\Delta u_{n}\right)^{\beta}\right)=-\psi_{n} v_{n+1}^{\lambda}, \\
\Delta\left(r_{n}\left(\Delta v_{n}\right)^{\alpha}\right)=\varphi_{n} u_{n+1}^{\eta},
\end{array}\right.
$$

which is again system of the form (3). Oscillation results of [5] for (3) assume

$$
\sum \frac{1}{r_{n}^{1 / \alpha}}=\infty, \quad \sum \frac{1}{q_{n}^{1 / \beta}}=\infty, \quad \sum \varphi_{n}<\infty, \quad \sum \psi_{n}=\infty
$$

that is (4) is not in the canonical form. Hence, to compare results of [5] and our oscillation criteria we have to apply results of [5] to (8), see Remark 6.

\section{Non-oscillatory solutions}

Throughout this and the next sections, we use the convention

$$
\sum_{i=n_{1}}^{n_{2}} u_{i}=0 \quad \text { if } n_{1}>n_{2}
$$

The aim of this section is to study non-oscillatory solutions of (1). If (S) has a solution $(x, y, z, w)$, then $(-x,-y,-z,-w)$ is a solution of (S), too. Hence, when studying the nonexistence conditions for non-oscillatory solutions, we can consider solutions such that $x_{n}>0$ for large $n$.

We start with the classification of non-oscillatory solutions of (S). This has been presented in [2] without the proof, so we formulate this statement including the proof. 
Lemma 2 Assume (H). Then any solution $(x, y, z, w)$ of system $(S)$ such that $x_{n}>0$ for large $n$ is one of the following types:

Type (a) $x_{n}>0, y_{n}>0, z_{n}>0, w_{n}>0$ for all large $n$,

Type (b) $x_{n}>0, y_{n}>0, z_{n}<0, w_{n}>0$ for all large $n$.

Proof Let $(x, y, z, w)$ be a non-oscillatory solution of (S). Assume that there exists a solution such that $y_{n}>0, z_{n}<0, w_{n}<0$ for large $n$. Since $\Delta z_{n}<0$, there exists $k>0$ such that $z_{n} \leq-k$ for large $n$. Using the summation of the second equation of system (S) we get

$$
y_{n}-y_{n_{0}}=\sum_{i=n_{0}}^{n-1} B_{i} z_{i}^{1 / \beta} \leq-k^{1 / \beta} \sum_{i=n_{0}}^{n-1} B_{i} .
$$

Passing $n \rightarrow \infty$, we get $\lim y_{n}=-\infty$, which is a contradiction.

Let there exist a solution so that $y_{n}<0, z_{n}>0, w_{n}>0$ for large $n$. Since $z$ is positive increasing there exists $k>0$ so that $z_{n} \geq k$ for large $n$. Summation of the second equation of system (S) leads to $\lim y_{n}=+\infty$, which is a contradiction with the fact $y_{n}<0$.

Let there exist a solution so that $y_{n}<0, z_{n}<0$ for large $n$. Since $y$ is negative decreasing there exists $k>0$ so that $y_{n} \leq-k$ for large $n$. By summation of the first equation of system (S) and passing $n \rightarrow \infty$, we get a contradiction.

The case when $z_{n}>0$ and $w_{n}<0$ for large $n$ can be treated by the similar way by summation of the third equation of $(S)$.

Proposition 1 Assume $(H)$ and

$$
\sum_{n=n_{0}}^{\infty} d_{n}=\infty
$$

Then Equation (1) is oscillatory.

Proof In view of Lemma 2 we can assume without loss of generality that $x_{n}>0, y_{n}>0$ and $w_{n}>0$. Hence exists $k>0$ and $n_{0}>1$ such that $x_{n} \geq k$ for $n \geq n_{0}$. By summation of the fourth equation of system (S) we find that (9) leads to a contradiction with the positiveness of $w_{n}$.

Hence, under assumptions $(\mathrm{H})$, if (1) has a non-oscillatory solution, then

$$
\sum_{n=n_{0}}^{\infty} d_{n}<\infty
$$

We say that a solution $x$ of (1) is of type (a) (type (b)) if the corresponding solution $(x, y, z, w)$ of system (S) is of type (a) (type (b)).

In the next, we give sufficient conditions for the non-existence of both types of nonoscillatory solutions of (1). To this goal, the following lemma will be used.

Lemma 3 Let $k \in(0,1)$ and $\left\{w_{n}\right\}$ be a sequence such that $w_{n}>0$ and $\Delta w_{n}<0$. Then

$$
\sum_{n=1}^{\infty} \frac{-\Delta w_{n}}{w_{n}^{k}}<\infty
$$


Proof We have

$$
\frac{-\Delta w_{n}}{w_{n}^{k}} \leq \int_{w_{n+1}}^{w_{n}} \frac{1}{t^{k}} d t
$$

Summing this from $N$ to $\infty$

$$
\sum_{n=N}^{\infty} \frac{-\Delta w_{n}}{w_{n}^{k}} \leq \sum_{n=N}^{\infty} \int_{w_{n+1}}^{w_{n}} \frac{1}{t^{k}} d t \leq \int_{0}^{w_{N}} \frac{1}{t^{k}} d t<\infty
$$

The non-existence of solutions of type (a) is ensured by the following conditions.

Lemma 4 Equation (1) has no solution of type (a) if any of the following conditions hold:

(i)

$$
\sum_{n=n_{0}}^{\infty} d_{n}\left(\sum_{i=n_{0}}^{n+\tau-1} \frac{1}{c_{i}^{1 / \gamma}}\right)^{\lambda}=\infty
$$

(ii)

$$
\sum_{n=n_{0}}^{\infty} d_{n}\left(\sum_{i=n_{0}}^{n+\tau-1} \frac{1}{c_{i}^{1 / \gamma}}\left(\sum_{j=n_{0}}^{i-1} \frac{1}{b_{j}^{1 / \beta}}\right)^{1 / \gamma}\right)^{\lambda}=\infty
$$

(iii) $\lambda<\alpha \beta \gamma$ and

$$
\sum_{n=n_{0}}^{\infty} d_{n}\left(\sum_{i=n_{0}}^{n+\tau-1} \frac{1}{c_{i}^{1 / \gamma}}\left(\sum_{j=n_{0}}^{i-1} \frac{1}{b_{j}^{1 / \beta}}\left(\sum_{k=n_{0}}^{j-1} \frac{1}{a_{k}^{1 / \alpha}}\right)^{1 / \beta}\right)^{1 / \gamma}\right)^{\lambda}=\infty .
$$

Proof Let $(x, y, z, w)$ be a type (a) solution of system (S), i.e., all components of the solution are positive. Since $z$ is positive increasing, there exists $k>0$ such that $z_{n}^{1 / \beta} \geq k$ for large $n$, say $n \geq n_{0}$. From the first and the second equations of system (S) we get

$$
x_{j} \geq \sum_{i=n_{0}}^{j-1} C_{i} y_{i}^{1 / \gamma}, \quad y_{j} \geq \sum_{i=n_{0}}^{j-1} B_{i} z_{i}^{1 / \beta} \geq k \sum_{i=n_{0}}^{j-1} B_{i},
$$

so

$$
x_{j} \geq \sum_{n=n_{0}}^{j-1} C_{n}\left(\sum_{k=n_{0}}^{n-1} B_{k} z_{k}^{1 / \beta}\right)^{1 / \gamma} \geq k^{1 / \gamma} \sum_{n=n_{0}}^{j-1} C_{n}\left(\sum_{k=n_{0}}^{n-1} B_{k}\right)^{1 / \gamma} .
$$

Let (11) or (12) hold. By summation of the fourth equation of system (S) and using (14) we get

$$
-w_{n}+w_{n_{0}}=\sum_{i=n_{0}}^{n-1}-\Delta w_{i} \geq k^{\lambda / \beta \gamma} k^{\lambda / \gamma} \sum_{i=n_{0}}^{n-1} D_{i}\left(\sum_{j=n_{0}}^{i+\tau-1} C_{j}\left(\sum_{k=n_{0}}^{j-1} B_{k}\right)^{1 / \gamma}\right)^{\lambda} .
$$

Passing $n \rightarrow \infty$ we get the contradiction with the boundedness of $w$. 
Let condition (iii) hold. Taking into account that $w_{n}$ is positive and decreasing, we get by summation of the third equation of system $(\mathrm{S})$

$$
z_{j} \geq \sum_{i=n_{0}}^{j-1} A_{i} w_{i}^{1 / \alpha} \geq w_{j-1}^{1 / \alpha} \sum_{i=n_{0}}^{j-1} A_{i}
$$

Thus

$$
-\Delta w_{n}=D_{n} x_{n+\tau}^{\lambda} \geq D_{n}\left(\sum_{m=n_{0}}^{n+\tau-1} C_{m}\left(\sum_{k=n_{0}}^{m-1} B_{k}\left(w_{k-1}^{1 / \alpha} \sum_{i=n_{0}}^{k-1} A_{i}\right)^{1 / \beta}\right)^{1 / \gamma}\right)^{\lambda} .
$$

Hence

$$
\frac{-\Delta w_{n}}{w_{n-1}^{\lambda / \alpha \beta \gamma}} \geq D_{n}\left(\sum_{m=n_{0}}^{n+\tau-1} C_{m}\left(\sum_{k=n_{0}}^{m-1} B_{k}\left(\sum_{i=n_{0}}^{k-1} A_{i}\right)^{1 / \beta}\right)^{1 / \gamma}\right)^{\lambda} .
$$

Summing this inequality from $n_{0}$ to $\infty$ we have

$$
\sum_{n=n_{0}}^{\infty} \frac{-\Delta w_{n}}{w_{n-1}^{\lambda / \alpha \beta \gamma}} \geq \sum_{n=n_{0}}^{\infty} D_{n}\left(\sum_{i=n_{0}}^{n+\tau-1} C_{i}\left(\sum_{j=n_{0}}^{i-1} B_{j}\left(\sum_{k=n_{0}}^{j-1} A_{k}\right)^{1 / \beta}\right)^{1 / \gamma}\right)^{\lambda} .
$$

By Lemma 3 the expression on the left side is finite, which is a contradiction with (13).

The non-existence of solutions of type (b) is ensured by the following conditions.

Lemma 5 Let (10) hold. Equation (1) has no solution of type (b) if any of the following conditions hold:

(i)

$$
T:=\sum_{n=n_{0}}^{\infty} \frac{1}{a_{n}^{1 / \alpha}}\left(\sum_{k=n}^{\infty} d_{k}\right)^{1 / \alpha}=\infty
$$

(ii) $T<\infty$ and

$$
\sum_{n=n_{0}}^{\infty} \frac{1}{b_{n}^{1 / \beta}}\left(\sum_{k=n}^{\infty} \frac{1}{a_{k}^{1 / \alpha}}\left(\sum_{i=k}^{\infty} d_{i}\right)^{1 / \alpha}\right)^{1 / \beta}=\infty
$$

(iii) $\lambda<\alpha \beta \gamma, T<\infty$ and

$$
\sum_{n=n_{0}}^{\infty} \frac{1}{b_{n}^{1 / \beta}}\left(\sum_{k=n_{0}}^{n+\tau-1} \frac{1}{c_{k}^{1 / \gamma}}\right)^{\lambda /(\alpha \beta)}\left(\sum_{k=n}^{\infty} \frac{1}{a_{k}^{1 / \alpha}}\left(\sum_{i=k}^{\infty} d_{i}\right)^{1 / \alpha}\right)^{1 / \beta}=\infty .
$$

Proof Let $(x, y, z, w)$ be a solution of (S) satisfying $x_{n}>0, y_{n}>0, z_{n}<0, w_{n}>0$. Since the components $w$ and $-z$ are positive and decreasing, we have

$$
\lim _{n \rightarrow \infty} w_{n}=w_{\infty}, \quad w_{\infty} \geq 0, \quad \lim _{n \rightarrow \infty} z_{n}=z_{\infty}, \quad z_{\infty} \leq 0 .
$$


By summation of the fourth equation of $(S)$ we have

$$
w_{n}=w_{\infty}+\sum_{k=n}^{\infty} D_{k} x_{k+\tau}^{\lambda} \geq x_{n+\tau}^{\lambda} \sum_{k=n}^{\infty} D_{k}
$$

If (i) holds, then by summation of the third equation of (S)

$$
z_{m} \geq z_{n_{0}}+x_{n_{0}+\tau}^{\lambda / \alpha} \sum_{n=n_{0}}^{m-1} A_{n}\left(\sum_{k=n}^{\infty} D_{k}\right)^{1 / \alpha}
$$

which gives a contradiction with the boundedness of $z$.

Assume (ii). Then

$$
-z_{n}=-z_{\infty}+\sum_{i=n}^{\infty} A_{i} w_{i}^{1 / \alpha} \geq x_{n+\tau}^{\lambda / \alpha} \sum_{i=n}^{\infty} A_{i}\left(\sum_{j=i}^{\infty} D_{j}\right)^{1 / \alpha}
$$

Using this and the fact that $y$ is positive decreasing, we get

$$
y_{n_{0}}=y_{n}+\sum_{k=n_{0}}^{n-1} B_{k}\left(-z_{k}\right)^{1 / \beta} \geq \sum_{k=n_{0}}^{n-1} B_{k}\left(\sum_{i=k}^{\infty} A_{i}\left(\sum_{j=i}^{\infty} D_{j}\right)^{1 / \alpha}\right)^{1 / \beta},
$$

which leads to a contradiction with (16).

Assume (iii). From the first equation of system (S) we have for large $n$

$$
x_{n} \geq y_{n-1}^{1 / \gamma} \sum_{i=n_{0}}^{n-1} C_{i}
$$

Thus using the second equation of system (S) and (18)

$$
-\Delta y_{n}=B_{n}\left(-z_{n}\right)^{1 / \beta} \geq B_{n} y_{n+\tau-1}^{\lambda / \alpha \beta \gamma}\left(\sum_{i=n_{0}}^{n+\tau-1} C_{i}\right)^{\lambda / \alpha \beta}\left(\sum_{i=n}^{\infty} A_{i}\left(\sum_{j=i}^{\infty} D_{j}\right)^{1 / \alpha}\right)^{1 / \beta}
$$

So

$$
\frac{-\Delta y_{n}}{y_{n+\tau-1}^{\lambda / \alpha \beta \gamma}} \geq B_{n}\left(\sum_{i=n_{0}}^{n+\tau-1} C_{i}\right)^{\lambda / \alpha \beta}\left(\sum_{i=n}^{\infty} A_{i}\left(\sum_{j=i}^{\infty} D_{j}\right)^{1 / \alpha}\right)^{1 / \beta} .
$$

Since $\alpha \beta \gamma>\lambda$ we get by Lemma 3

$$
\infty>\sum_{n=n_{0}}^{\infty} \frac{-\Delta y_{n}}{y_{n+\tau-1}^{\lambda / \alpha \beta \gamma}} \geq \sum_{n=n_{0}}^{\infty} B_{n}\left(\sum_{i=n_{0}}^{n+\tau-1} C_{i}\right)^{\lambda / \alpha \beta}\left(\sum_{i=n}^{\infty} A_{i}\left(\sum_{j=i}^{\infty} D_{j}\right)^{1 / \alpha}\right)^{1 / \beta}
$$

which is a contradiction.

Remark 3 The condition $(\mathrm{H})$ is not needed in Lemmas 4 and 5. 
Remark 4 (i) Lemmas 4 and 5 can be viewed as a discrete counterpart of similar results for differential systems of the $n$ th-order, see [14, Propositions 4.1, 4.5].

(ii) Oscillation criteria established in [2] are based on a different approach than that applied here, namely by comparing (1) with certain first- and second-order difference equations whose oscillatory properties are known. Comparing conditions for the nonexistence of solutions of types (a) and (b), part (iii) of Lemmas 4 and 5 extends Corollaries 2.2 and 2.1 in [2], respectively, where it is assumed that $\tau \leq 0$ and $(\mathrm{H})$. Moreover, assuming $(\mathrm{H})$, part (ii) of Lemmas 4 and 5 can be obtained from Theorems 2.6 and 2.4 in [2], respectively, but our proofs are different.

Combining conditions in Lemmas 4 and 5, we get oscillation criteria in case where the operator in (1) is in the canonical form. This, together with the application of the cyclic permutation method, will form the content of the following two sections.

\section{Oscillation criteria}

In this section, we establish oscillation criteria for (1) which are based on conditions for the non-existence of the non-oscillatory solutions given in the previous section.

First we discuss conditions (12) and (15). Assume (H), (10) and consider the double series

$$
P=\sum_{n=1}^{\infty} d_{n}\left(\sum_{k=1}^{n} \frac{1}{c_{k}^{1 / \gamma}}\right)^{\lambda}, \quad T=\sum_{n=1}^{\infty} \frac{1}{a_{n}^{1 / \alpha}}\left(\sum_{k=n}^{\infty} d_{k}\right)^{1 / \alpha} .
$$

If $P=\infty$ and $T=\infty$, then by Lemmas $2,4,5$, Equation (1) with $\tau \geq 1$ is oscillatory.

In a special case when $\alpha=\gamma=\lambda=1$ and $a_{n}=c_{n}$ we have $P=\infty$ if and only if $T=\infty$.

The interesting case occurs when $\alpha=\lambda \neq 1$ or $\alpha \neq \lambda$. The problem of comparison of conditions (12) and (15) leads to the problem of a change of summation for double series. This problem has been investigated for the case $\alpha=\lambda$ and $\alpha \neq \lambda$ in [17, 18], respectively.

For brevity, denote the following cases of parameters $\alpha, \lambda$ :

(C1) $\alpha>\lambda$ or $\alpha=\lambda \geq 1$;

(C2) $\alpha<\lambda$ or $\alpha=\lambda \leq 1$.

Put

$$
S=\sum_{n=1}^{\infty} d_{n}\left(\sum_{k=1}^{n} \frac{1}{a_{k}^{1 / \alpha}}\right)^{\lambda} .
$$

In cases $(\mathrm{C} 1)$ and $(\mathrm{C} 2)$ the following change of summation holds.

\section{Lemma $6([17,18])$}

(i) Assume case (C1). If $S=\infty$, then $T=\infty$.

(ii) Assume case (C2). If $T=\infty$, then $S=\infty$.

Remark 5 Observe that the opposite implications in Lemma 6 in general need not hold. For example, choosing

$$
S=\sum_{n=1}^{\infty} \frac{1}{n(n-1)}\left(\sum_{k=1}^{n} 1\right)^{\lambda} \text { and } T=\sum_{n=1}^{\infty}\left(\sum_{k=n}^{\infty} \frac{1}{k(k-1)}\right)^{1 / \alpha}
$$

we have $S=\infty$ and $T<\infty$ for $\lambda \geq 1$ and $\alpha<1$; the opposite case holds for $\lambda<1$ and $\alpha \geq 1$. 
Using Lemma 6 we obtain the following result.

Theorem 3 Assume (H) and (10). Equation (1) with $\tau \geq 1$ is oscillatory if any of the following conditions holds:

(i) Case (C1), $P=\infty$ and

$$
\liminf \frac{c_{n}^{1 / \gamma}}{a_{n}^{1 / \alpha}}>0
$$

(ii) Case (C2), $T=\infty$ and

$$
\limsup \frac{c_{n}^{1 / \gamma}}{a_{n}^{1 / \alpha}}<\infty
$$

Proof Claim (i). Clearly, condition $P=\infty$ implies the validity of (11) for any $\tau \geq 1$. Hence, by Lemma 4, Equation (1) with $\tau \geq 1$ has no type (a) solution. By comparison theorem for series and in view of (19), we have $S=\infty$. Using Lemma 6 we get $T=\infty$. By Lemma 5 Equation (1) has no type (b) solutions. Now, the conclusion follows from Lemma 2. Claim (ii) can be proved by a similar way.

In the general case, when Theorem 3 cannot be applied, by Lemma 4, part (ii) and Lemma 5, parts (i), (ii) the following result holds.

Theorem 4 ([2, Theorem 2.10]) Assume (H), (10) and $\tau \in \mathbb{Z}$. If (12) and either (15) or (16) hold, then Equation (1) is oscillatory.

In the sublinear case, this result can be improved using part (iii) of Lemmas 4 and 5 as follows.

Theorem 5 Assume $\lambda<\alpha \beta \gamma,(H)$, (10) and $\tau \in \mathbb{Z}$. If (13) and either (15) or (17) hold, then Equation (1) is oscillatory.

Remark 6 Theorems 3, 4, 5 can be compared with the results of [5] using coupled system (8). Application of Theorem 1 or Theorem 2' of [5] to system (8) leads to conditions (11), (15) or (13), (15), respectively. Observe that Theorem $4^{\prime}$ of [5] ensures oscillation of (8) provided $\lambda<1,(13)$ and certain additional assumptions on $\alpha, \beta, \gamma$.

The following examples illustrate our results and show that conditions in Theorem 5 are optimal.

Example 2 Consider the equation

$$
\Delta\left(\Delta^{3} x_{n}\right)^{\alpha}+d_{n} x_{n+\tau}^{\lambda}=0,
$$

where $\tau \geq 1$ and (10) holds. Then

$$
P=\sum_{n=1}^{\infty} n^{\lambda} d_{n}, \quad T=\sum_{n=1}^{\infty}\left(\sum_{k=n}^{\infty} d_{k}\right)^{1 / \alpha}
$$


and by Theorems 3 and 5 we get that Equation (20) is oscillatory if any of the following conditions is satisfied

(i) $\lambda<\alpha$ or $\alpha=\lambda \geq 1, P=\infty$;

(ii) $\lambda>\alpha$ or $\alpha=\lambda \leq 1, T=\infty$;

(iii) $\lambda<\alpha, \sum_{n=1}^{\infty} n^{3 \lambda} d_{n}=\infty, T<\infty$ and

$$
\sum_{n=1}^{\infty} n^{\lambda / \alpha} \sum_{j=n}^{\infty}\left(\sum_{k=j}^{\infty} d_{k}\right)^{1 / \alpha}=\infty
$$

The claim (iii) of Example 2 is not true for $\alpha=\lambda=1$ as the next example shows.

Example 3 Consider the Euler-type difference equation

$$
\Delta^{4} x_{n}+\frac{24}{(n+3)(n+4)(n+5)(n+6)} x_{n+3}=0 \quad(n \geq 2) .
$$

One can check that $x_{n}=n(n+1)(n+2)(n+3)$ is a positive solution of $(21)$ and $\sum_{n=1}^{\infty} n^{3} d_{n}=$ $\infty$.

Another oscillation criteria can be obtained using the cyclic permutation described in Lemma 1 and Theorem 2. For instance, in the case when

$$
\sum_{n=n_{0}}^{\infty} a_{n}^{-1 / \alpha}=\infty, \quad \sum_{n=n_{0}}^{\infty} b_{n}^{-1 / \beta}<\infty, \quad \sum_{n=n_{0}}^{\infty} c_{n}^{-1 / \gamma}=\sum_{n=n_{0}}^{\infty} d_{n}=\infty
$$

we can apply Theorems 3-5 to Equation (R2). We show the application of Theorem 5 to a special case of (1) in the next section.

\section{Applications}

Consider equation

$$
\Delta^{2}\left(b_{n}\left(\Delta^{2} x_{n}\right)^{\beta}\right)+d_{n} x_{n+\tau}^{\lambda}=0,
$$

where $\tau \in \mathbb{Z}$ and

$$
\sum_{n=n_{0}}^{\infty} \frac{1}{b_{n}^{1 / \beta}}<\infty \quad \text { and } \quad \sum_{n=n_{0}}^{\infty} d_{n}=\infty
$$

Then the cyclic permutated Equation (R2) to (22) is

$$
\Delta^{2}\left(\frac{1}{d_{n}^{1 / \lambda}}\left(\Delta^{2} z_{n}\right)^{1 / \lambda}\right)+\frac{1}{b_{n+\tau}^{1 / \beta}} z_{n+\tau}^{1 / \beta}=0
$$

whose difference operator is in the canonical form. In Equation (24), we have $\alpha=1, \beta=$ $1 / \lambda, \gamma=1, \lambda=1 / \beta$, thus the condition $\lambda<\alpha \beta \gamma$ reads $\lambda<\beta$ and the series $P$ and $T$ as

$$
\bar{P}=\sum_{n=n_{0}}^{\infty}\left(\frac{n}{b_{n+\tau}}\right)^{1 / \beta}, \quad \bar{T}=\sum_{n=n_{0}}^{\infty} \sum_{k=n}^{\infty} \frac{1}{b_{k+\tau}^{1 / \beta}}=\sum_{n=n_{0}}^{\infty} \frac{n-n_{0}+1}{b_{n+\tau}^{1 / \beta}} .
$$


Since $\lim _{n \rightarrow \infty} \frac{n+\tau}{n}=1$, we have $\bar{P}=\infty$ if and only if

$$
\tilde{P}=\sum_{n=n_{0}}^{\infty}\left(\frac{n}{b_{n}}\right)^{1 / \beta}=\infty \text {. }
$$

Similarly, since $\lim _{n \rightarrow \infty} \frac{n+\tau}{n-n_{0}+1}=1$, we get $\bar{T}=\infty$ if and only if

$$
\tilde{T}=\sum_{n=n_{0}}^{\infty} \frac{n}{b_{n}^{1 / \beta}}=\infty .
$$

Observe that if $\beta \geq 1$ and $\tilde{P}=\infty$, then $\tilde{T}=\infty$, while if $\beta \leq 1$ and $\tilde{T}=\infty$, then $\tilde{P}=\infty$. Hence, under these assumptions both series $\tilde{P}, \tilde{T}$ are divergent and Equation (22) with $\tau \geq 1$ is oscillatory by Theorem 2 .

Applying Theorem 5 to Equation (24) and using Theorem 2, we get the following result.

Corollary 1 Assume (23) and $\lambda<\beta, \tau \in \mathbb{R}$. If

$$
\sum_{n=n_{0}}^{\infty} \frac{1}{b_{n+\tau}^{1 / \beta}}\left(\sum_{j=n_{0}}^{n+\tau-1} j^{\lambda} d_{j}\right)^{1 / \beta}=\infty
$$

and either $\tilde{T}=\infty$ or

$$
\sum_{n=n_{0}}^{\infty} n^{\lambda / \beta} d_{n}\left(\sum_{k=n}^{\infty} \frac{k}{b_{k}^{1 / \beta}}\right)^{\lambda}=\infty,
$$

then Equation (22) is oscillatory.

Remark 7 Corollary 1 completes the oscillation criteria for Equation (22) with $\tau=3$ given in $[10,12]$ where instead of the condition $\sum d_{n}=\infty$ it is assumed that both series $\tilde{P}, \tilde{T}$ are divergent or convergent, respectively.

\section{Competing interests}

The authors declare that they have no competing interests.

\section{Authors' contributions}

Both authors contributed equally to the manuscript and read and approved the final draft.

\section{Acknowledgements}

Zuzana Došlá was supported by the Grant P201/10/1032 of the Czech Grant Agency, and Jana Krejčová was supported by the Grant MUNI/A/0964/2009. The authors thank the referees for their useful comments.

Received: 27 December 2011 Accepted: 18 June 2012 Published: 2 July 2012

\section{References}

1. Agarwal, RP, Grace, SR, Wong, PJY: Oscillatory behavior of fourth order nonlinear difference equations. N.Z. J. Math. 36, 101-111 (2007)

2. Agarwal, RP, Grace, SR, Manojlović, JV: On the oscillatory properties of certain fourth order nonlinear difference equations. J. Math. Anal. Appl. 322, 930-956 (2006)

3. Agarwal, RP, Manojlović, JV: Asymptotic behavior of positive solutions of fourth order nonlinear difference equations. Ukr. Math. J. 60, 6-28 (2008)

4. Agarwal, RP, Manojlović, JV: Asymptotic behavior of nonoscillatory solutions of fourth order nonlinear difference equations. Dyn. Contin. Discrete Impuls. Syst., Ser. A, Math. Anal. 16, 155-174 (2009) 
5. Marini, M, Matucci, S, Řehák, P: Oscillation of coupled nonlinear discrete systems. J. Math. Anal. Appl. 295, 459-472 (2004)

6. Popenda, J, Schmeidel, E: On the solution of fourth order difference equations. Rocky Mt. J. Math. 25(4), 1485-1499 (1995)

7. Smith, B, Taylor, WE: Oscillatory and asymptotic behavior of certain fourth order difference equations. Rocky Mt. J. Math. 16(2), 403-406 (1986)

8. Thandapani, E, Graef, JR: Oscillatory and asymptotic behavior of fourth order nonlinear delay difference equations. Fasc. Math. 31, 23-36 (2001)

9. Thandapani, E, Pandian, S, Dhanasekaran, R, Graef, J: Asymptotic results for a class of fourth order quasilinear difference equations. J. Differ. Equ. Appl. 13(12), 1085-1103 (2007)

10. Thandapani, E, Vijaya, M: Oscillatory and asymptotic behavior of fourth order quasilinear difference equations. Electron. J. Qual. Theory Differ. Equ. 2009(64), 1-15 (2009)

11. Thandapani, E, Selvaraj, B: Oscillations of fourth order quasilinear difference equations. Fasc. Math. 37, 109-119 (2007)

12. Thandapani, E, Selvaraj, B: Oscillatory and nonoscillatory behavior of fourth order quasilinear difference equations. Far East J. Appl. Math. 17(3), 287-307 (2004)

13. Thandapani, E, Arockiasamy, IM: On fourth order nonlinear oscillations of difference equations. Comput. Math. Appl. 42, 357-368 (2001)

14. Kusano, T, Naito, M, Wu, F: On the oscillation of solutions of 4-dimensional Emden-Fowler differential systems. Adv. Math. Sci. Appl. 11(2), 685-719 (2001)

15. Chanturia, TA: On oscillatory properties of systems of nonlinear ordinary differential equations. Proc. I. Vekua Inst. Appl. Math. 14, 163-204 (1983) (in Russian)

16. Došlá, Z, Krejčová, J: Nonoscillatory solutions of the four-dimensional difference system. Electron. J. Qual. Theory Differ. Equ. Proc. 9th Coll. QTDE 4, 1-11 (2011)

17. Cecchi, M, Došlá, Z, Marini, M, Vrkoč, I: Summation inequalities and half-linear difference equations. J. Math. Anal. Appl. 302, 1-13 (2005)

18. Cecchi, M, Došlá, Z, Marini, M, Vrkoč, I: Asymptotic properties for half-linear difference equation. Math. Bohem. 131(4), 347-363 (2006)

doi:10.1186/1687-1847-2012-99

Cite this article as: Došlá and Krejčová: Oscillation of a class of the fourth-order nonlinear difference equations. Advances in Difference Equations 2012 2012:99.

\section{Submit your manuscript to a SpringerOpen ${ }^{\circ}$ journal and benefit from:}

- Convenient online submission

- Rigorous peer review

- Immediate publication on acceptance

- Open access: articles freely available online

- High visibility within the field

- Retaining the copyright to your article 